\title{
BMJ Open Alcoholic Chlorhexidine or Alcoholic Iodine Skin Antisepsis (ACAISA): protocol for cluster randomised controlled trial of surgical skin preparation for the prevention of superficial wound complications in prosthetic hip and knee replacement surgery
}

\author{
T N Peel, ${ }^{1,2}$ A C Cheng, ${ }^{3,4}$ K L Buising, ${ }^{5}$ M M Dowsey, ${ }^{1,2}$ P F M Choong ${ }^{1,2}$
}

To cite: Peel TN, Cheng AC, Buising KL, et al. Alcoholic Chlorhexidine or Alcoholic lodine Skin Antisepsis (ACAISA): protocol for cluster randomised controlled trial of surgical skin preparation for the prevention of superficial wound complications in prosthetic hip and knee replacement surgery. BMJ Open 2014;4:e005424. doi:10.1136/bmjopen-2014005424

- Prepublication history for this paper is available online. To view these files please visit the journal online (http://dx.doi.org/10.1136/ bmjopen-2014-005424).

Received 8 April 2014 Revised 22 April 2014 Accepted 23 April 2014

CrossMark

For numbered affiliations see end of article.

Correspondence to

Dr T N Peel;

tnpeel@unimelb.edu.au

\section{ABSTRACT}

Introduction: Wound complications following arthroplasty are associated with significant impact on the patient and healthcare system. Skin cleansing prior to surgical incision is a simple and effective method to prevent wound complications however, the question of which agent is superior for surgical skin antisepsis is unresolved.

Methods and analysis: This cluster randomised controlled trial aims to compare the incidence of superficial wound complications in patients undergoing elective prosthetic hip or knee replacement surgery receiving surgical skin antisepsis with either: $0.5 \%$ chlorhexidine gluconate (CHG) in $70 \%$ alcohol or $10 \%$ povidone in $70 \%$ alcohol. The trial will be conducted at an Australian tertiary, university affiliated hospital over a 3-year period involving 750 participants. Participants will be drawn from the surgical waiting list. Consent for this study will be 'opt-out' consent. On a given day, all eligible participants will have skin preparation either with $0.5 \%$ chlorhexidine in $70 \%$ alcohol or $10 \%$ povidone iodine in $70 \%$ alcohol. The primary outcome is superficial wound complications (comprised of superficial incisional surgical site infections (SSI) and/ or prolonged wound ooze) in the first 30 days following prosthetic joint replacement surgery. Secondary outcomes will include the incidence of wound complications according to the joint replaced, assessment of the causative agents of SSI and costeffectiveness analysis. The primary analysis is an intention-to-treat analysis including all participants who undergo randomisation and will be performed at the individual level taking into account the clustering effect. Ethics and dissemination: The study design and protocol was reviewed and approved by the St Vincent's Hospital Human Research Ethics Committee (HREC-A 016/14 10/3/2014). Study findings will be disseminated in the printed media, and learned forums. A written lay summary will be available to study participants on request.

Trial registration number: The trial has been registered with the Australian New Zealand Clinical Trials Registry (ANZCTR): ACTRN12614000177651.

\section{BACKGROUND}

Following prosthetic joint replacement surgery, superficial wound complications (SWC), comprised of superficial surgical site infections (SSI) and prolonged wound ooze, have been consistently linked to prosthetic joint infections; increasing the risk of these infections by $55-60 \% .^{1-3}$ In Australia the conservative estimate of annual direct healthcare costs of treating SWC following prosthetic joint replacement surgery is $\$ 34$ million. $^{4-6}$ SSI, such as superficial incisional SSI and organ/space SSI (such as prosthetic joint infections) may occur in up to $15 \%$ of patients undergoing surgery and are associated with significant patient suffering including the need for prolonged hospitalisation, further surgical procedures, prolonged antibiotic use and negative impact on quality of life. ${ }^{5} 78$ There is also a significant ecological impact of these infections with the generation of increasingly resistant microorganisms as a consequence of antibiotic therapy. ${ }^{9-11}$ The economic burden of SSI is also substantial; SSI increase the direct 
healthcare costs by more than $300 \% .{ }^{6}$ Prevention of SWC may substantially decrease morbidity and mortality, improve patient outcomes and reduce the economic burden to the healthcare system. ${ }^{6}$

Human skin is colonised with millions of bacteria and is the major source of infecting pathogens, including Staphylococcus spp. ${ }^{78} 12$ The number of contaminating microorganisms required to produce infection is low, particularly in the setting of implantation of prosthetic material (such as a prosthetic joint surgery). ${ }^{13}{ }^{14}$ Surgical skin antisepsis is a simple and effective strategy to decontaminate patient's skin prior to surgical incision. ${ }^{15} 16$ The three main agents commonly used for surgical skin antisepsis are chlorhexidine gluconate (CHG), iodophors or alcohol. ${ }^{15}$ Alcohol is frequently combined with CHG or iodophors to optimise the activity of the surgical skin antisepsis. ${ }^{8} 17$ These agents are inexpensive and are well tolerated; adverse reactions, predominantly mild skin reactions, are rare and occur in $<1 \%$ of patients. ${ }^{16}$ Current international guidelines, including National Institute for Health and Care Excellence (NICE) and Centers for Disease Control and Prevention (CDC), recommend use of either iodophors or CHG as surgical skin antisepsis. ${ }^{78} 1819$

There is a paucity of well-controlled studies examining the impact of surgical skin antisepsis on SWC. ${ }^{8}$ In particular there are no randomised controlled trials comparing $\mathrm{CHG} /$ alcohol and iodophor/alcohol combination and the question of which agent is superior is unresolved. ${ }^{15}$ Current recommendations for surgical skin antisepsis are drawn from studies including a seminal, large, multicentre randomised clinical trial by Darouiche et $a l^{16}$ comparing skin preparation with $2 \%$ CHG plus $70 \%$ isopropyl alcohol to $10 \%$ povidone iodine in clean-contaminated surgery. This study demonstrated a reduction in SSI with the use of CHG plus alcohol compared to povidone iodine. However, this study did not include a comparator arm with povidone iodine combined with alcohol therefore it is difficult to attribute the observed difference to chlorhexidine or alcohol (or both in combination). ${ }^{16}$ Authors from a Cochrane Review concluded "there was insufficient evidence from randomised trials to support or refute the use of one antiseptic over another" and called for further research. ${ }^{7}$ Similar conclusions have been drawn in other systematic reviews and meta-analyses. ${ }^{15}{ }^{20} \mathrm{In}$ addition, no data exists on the cost effectiveness of preoperative skin antisepsis. ${ }^{7}$

Prosthetic joint surgery is an important setting to study the efficacy of surgical skin antisepsis. It represents high volume, high-cost surgery in which SWC can have a devastating effect. In 2012, 90000 Australian patients underwent prosthetic joint surgery, representing an increase of $40 \%$ over the previous 10 years and this is expected to double again by $2020 .{ }^{21}{ }^{22}$ Our pilot data have shown that SWC occurred in $9 \%$ of patients undergoing prosthetic hip and knee replacement surgery and $30 \%$ required re-admission to acute care hospital for management of the wound complication. Furthermore there was a $92 \%$ increased risk of subsequent prosthetic joint infection in patients with SWC. In multivariate analysis of risk factors for SWC, we showed that surgical skin antisepsis with $0.5 \%$ CHG in $70 \%$ alcohol was associated with a $60 \%$ increased risk of SWC compared with $10 \%$ povidone iodine ( $1 \%$ available iodine) in $70 \%$ alcohol. The association was particularly marked in the prosthetic hip replacement cohort with an $80 \%$ increased risk of SWC. ${ }^{23}$ The aim of this cluster randomised controlled trial is to compare the incidence of SWC in all patients undergoing elective prosthetic hip or knee replacement surgery receiving surgical skin antisepsis with either $0.5 \%$ CHG in $70 \%$ alcohol or $10 \%$ povidone iodine (with $1 \%$ available iodine) in $70 \%$ alcohol.

\section{METHODS AND ANALYSIS}

\section{Study centre}

St Vincent's Hospital Melbourne (SVHM) is a Victorian State Centre for joint replacement surgery and a leading academic orthopaedic department with a major focus on prosthetic joint surgery. The SVHM Department of Orthopaedics pursues a major research interest in predictors of outcomes after prosthetic joint surgery and maintains a comprehensive database of all lower limb primary arthroplasties performed since 1998. Extensive follow-up data to 12 years are also captured; the SVHM arthroplasty registry has a minimum of 12 months of follow-up data for $98 \%$ of patients undergoing prosthetic hip and knee replacement surgery. SVHM Orthopaedic Department is currently staffed by 15 orthopaedic surgeons performing over 700 elective prosthetic hip and knee replacements per year. All hospital postoperative care is performed according to the SVHM Hip or Knee Replacement Clinical Pathway. ${ }^{24}$

\section{Study design}

We propose a cluster randomised controlled trial comparing $0.5 \%$ CHG in $70 \%$ alcohol with $10 \%$ povidone iodine (with $1 \%$ available iodine) in $70 \%$ alcohol for surgical skin preparation in the prevention of SWCs in hip and knee prosthetic joint replacement surgery. The study will be conducted at SVHM over a 3-year period. As a prospective randomised controlled trial, the study strategy will be constructed and presented in accordance with the recommendations of the CONSORT statement. $^{25}$

\section{Intervention}

Study arm (A): surgical skin antisepsis with $0.5 \%$ CHG in $70 \%$ alcohol.

Study arm (B): surgical skin antisepsis with $10 \%$ povidone iodine ( $1 \%$ available iodine) in $70 \%$ alcohol.

\section{Recruitment of study participants}

Participant consent for this study is 'opt-out consent'. The study population will be drawn from patients on the 
waiting list for elective hip and knee replacement surgery at SVHM. Following procedural consent of the patient by the treating orthopaedic surgeon for the planned surgery (hip or knee joint replacement), the patient is placed on the Surgical Waiting List. Once a participant is placed on the waiting list, they will be sent the Patient Information Statement for Opt-Out Consent, which will detail the problem of SWC and the nature of the study. In addition the Patient Information Statement for Opt-Out Consent will explain the process to 'opt-out' of the study. The Project Research Officer will review the Patient Administration System demographic page to identify any participants with a primary language other than English. If such participant is identified, they will be sent the Patient Information Statement for Opt-Out Consent translated to their primary language by a certified translation service, in addition to the English version. The participants will be given a number of options to contact the Project Research Officer to opt-out, including phone, email and mailing address. In addition, patients may request to opt-out of the study at the time of presentation to the pre-admission clinic or on the day of surgery.

After the Patient Information Statement for Opt-Out Consent is sent to the participant, the Project Research Officer will review the medical record and any potential participant with a documented allergy to either study agent; will be excluded from the study.

\section{Randomisation}

In the opt-out approach, willingness to participate is presumed unless the participant communicates a choice not to participate in the research by the day of surgery. In this cluster randomised controlled trial, the unit of randomisation is the day of surgery. On a given day, all eligible participants will have skin preparation either with (A) $0.5 \%$ chlorhexidine in $70 \%$ alcohol or (B) $10 \%$ povidone iodine ( $1 \%$ available iodine) in $70 \%$ alcohol. Each cluster will be randomly assigned in a ratio of 1:1. Randomisation will be performed by a computergenerated random assignment sequence by a statistician and opaque, numbered, tamperproof envelopes containing assignment will be prepared in advance. In addition, the research team involved in the assessment or treatment of patients will have no role in the assignment process. The patients will be blinded to treatment allocation. We recognise that blinding of the operating surgeons to the assigned preventative strategy is not feasible given the different appearance of the CHG and povidone iodine; however, the operating surgeons will be blinded to the allocation until the day of surgery. In addition, the Project Research Officer will be blinded and data will be analysed on an intention-to-treat basis.

\section{Inclusion criteria}

All patients undergoing prosthetic hip or knee total joint replacement surgery at SVHM.
Exclusion criteria

- Patients $<18$ years of age.

- Patients with a documented allergy to chlorhexidine, alcohol or iodophors.

- Patients with a primary language other than English for which certified translation services for that specific language are not available.

- Patients undergoing arthroplasty surgery for traumatic fractured neck of femur.

- Patients undergoing insertion of a tumour endoprosthesis for bone and soft tissue tumours.

\section{Treatment protocol}

Surgical skin antisepsis will be applied in a consistent manner for both study arms (A and B) and will be consistent with international guidelines. ${ }^{7} 81819$ In addition the surgical skin antisepsis product will be single use and iodophor-impregnated incise drapes will not be used throughout the course of the study. ${ }^{8} 17$

\section{Outcome measures}

Patients will be followed for 30 days postprosthetic joint replacement surgery.

\section{Primary outcome measure}

The primary outcome measure is the incidence of postoperative SWC (defined below). ${ }^{23}$

\section{Secondary outcome measures}

The secondary outcome measures of the study will include:

- The incidence of SWC according to the joint replaced (knee or hip)

- The incidence of SSI and clinically significant wound ooze

- Assessment of the causative microorganisms of SSI

- The incidence of prosthetic joint infection (defined below)

- Undesirable adverse consequences from surgical skin antisepsis including toxicity and allergies

- Economic analysis including cost-effectiveness of surgical skin antisepsis

\section{Definitions}

Superficial wound complication

Defined if the participant develops a superficial SSI and/or clinically significant wound ooze within 30 days of the indexed prosthetic joint replacement surgery.

\section{Superficial SSI (modified from the CDC definition of SSI)}

- Infection occurs within 30 days after prosthetic joint surgery.

- Involves only skin or subcutaneous tissue of the incision.

- It meets at least one of the following:

- Purulent drainage from the superficial incision with or without laboratory confirmation, from the superficial incision. 
- Organisms isolated from an aseptically obtained culture of fluid or tissue from the superficial incision.

- At least one of the following signs or symptoms of infection: pain or tenderness, localised swelling, redness or heat and superficial incisions deliberately opened by surgeon, unless incision is culture negative.

- Diagnosis of a superficial incisional SSI by the surgeon or attending physician. ${ }^{8} 26$

\section{Clinically significant wound ooze}

- Occurs within 30 days after prosthetic joint surgery.

- It meets at least one of the following:

- There is documented drainage from the surgical incision that required intervention, such as superficial surgical debridement.

- The documented drainage leads to deviation from normal care as per the SVHM arthroplasty clinical pathway, such as delayed discharge from hospital or readmission. ${ }^{2} 3242728$

\section{Prosthetic joint infection (organ/space SSI)—modified} from the Musculoskeletal Infection Society definition for periprosthetic joint infection

- Occurs within 365 days from prosthetic hip or knee replacement surgery.

- It meets at least one of the following criteria:

- Presence of a sinus tract in direct communication with the prosthetic joint;

$-\geq 1$ indistinguishable microorganism/s cultured from $\geq 2$ aseptically obtained tissue or fluid samples (including intra-operative tissue cultures or joint aspirate) taken from the affected prosthetic joint.

- Four of the following six criteria exist:

- Elevated serum $\mathrm{C}$ reactive protein concentration $>5 \mathrm{mg} / \mathrm{mL}$;

- Elevated synovial leucocyte count $\left(>1.7 \times 10^{3} / \mu \mathrm{L}\right)$;

- Elevated synovial neutrophil (polymorphonuclear leucocytes; PMN) percentage ( $>65 \%$ PMN);

- Presence of purulence in the affected joint as documented in the operative notes;

- Isolation of a microorganism in one culture of periprosthetic tissue or fluid;

- Histological evidence of acute inflammation as per the pathology report. ${ }^{8} 26$ 29-32

\section{Data collection}

The processes for identifying outcomes will be conducted by the Project Research Officer who will be blinded to the intervention allocation. The Project Research Officer will collect the baseline demographic data on all study participants including sex, age, comorbidities and operation details. Participant information will be stored in a reidentifable format for the purposes of conducting phone follow-up and medical record review follow-up. Information will be stored both electronically and in paper copy during and after the research study. In order to maintain complete confidentiality and security, all files will be password protected, on a computer within the Orthopaedic Department at SVHM, which is a locked facility, and all paper documents stored in a locked cabinet within the department. Only researchers listed within this study will have access to these files. Information will be stored for 15 years as required by law.

In keeping with the institutional Human Research Ethics Committee (HREC) approval, the study will be conducted in accordance with the ethical and research arrangements of SVHM and relevant legislation and regulations. In addition, any serious or unexpected adverse effects on participants or any unforeseen events that might affect continued ethical acceptability of the project will be immediately reported to the HREC. Further, in keeping with the conditions of approval stipulated by the HREC, an annual progress reports and a final report will be provided to the HREC.

\section{Sample size}

From the pilot data the proportion of patients with SWC was $27 \%$ in the $0.5 \%$ chlorhexidine $/ 70 \%$ alcohol group versus $8 \%$ of patients in the $10 \%$ povidone iodine $(1 \%$ available iodine) $/ 70 \%$ alcohol group. On multivariate analysis there was an $80 \%$ reduction in the risk of SWC in the group receiving $10 \%$ povidone iodine $(1 \%$ available iodine) in $70 \%$ alcohol surgical skin antisepsis (OR $0.20,95 \%$ CIs 0.06 to 0.67$).^{23}$ The sample size estimation for this study is based on a minimally clinically significant difference of $9 \%$ (based on the upper limit of the 95\% CI of the pilot study) and includes the following parameters: (1) $\alpha$ value $=0.05$, two-sided; (2) power $=80 \%$; (3) expected rates of the primary outcome (defined above) at 30 days post-prosthetic hip or knee replacement surgery of $27 \%$ for $0.5 \%$ CHG in $70 \%$ alcohol and $18 \%$ for $10 \%$ povidone iodine ( $1 \%$ available iodine) in $70 \%$ alcohol. The sample size required in each of the two (equally sized) groups is 359 patients for individual randomisation. ${ }^{23}$ Previous published data suggests that the intracluster correlation coefficient is very small (median ICC <0.01) between infections in patients in the same surgical day, and therefore the design effect allowing for clustering is minimal. ${ }^{33-35}$ Based on the SVHM arthroplasty registry 12-month loss to follow-up rate of $2 \%$, we will aim to recruit 750 patients in total. At SVHM, over 700 prosthetic joint replacements are performed each year therefore recruitment will be completed within the first 2 years of the study.

\section{Epidemiological analysis}

Although the intervention is implemented at the day of surgery level (the cluster), the analysis will be performed at the individual level but will take into account the clustering effect. The primary analysis is an intention to treat analysis including all participants who underwent randomisation. The OR of SSI associated with the treatment arm compared with the control arm will be 
estimated using the generalised estimation equations accounting for potential clustering in the unit of randomisation, adjusting a priori for the joint replaced, gender, age group and presence of rheumatoid arthritis; these are all factors known to impact on the risk of infection. All reported $p$ values will be two-tailed and for each analysis $\mathrm{p}<0.05$ will be considered statistically significant when using Huber-White robust SE.

\section{Economic analysis}

Costs will be compared between groups using a Poisson regression, which assumes that costs are log-normally distributed. We will apply health economic modelling to estimate the potential cost effectiveness of $0.5 \%$ CHG in $70 \%$ alcohol compared with $10 \%$ povidone iodine in $70 \%$ alcohol. A decision analysis will be used to compare the downstream consequences of surgical skin antisepsis. ${ }^{36}$ Markov and life-tabling techniques will allow for the modelling of outcomes beyond 1 year and will include $5 \%$ discounting of estimated future costs and health gains. ${ }^{37} 38$

\section{ETHICS AND DISSEIMINATION}

This project is due for completion 3 years after the start. We expect that soon after the completion of the project, important findings will be readily translated into practice through dissemination in the printed and electronic media, and at learned forums. A written lay summary of the results will be available to study participants on request.

\section{Expected outcomes}

This proposal provides new knowledge to the field of prevention of SWC in patients undergoing prosthetic joint-replacement surgery, addressing questions raised in the recent Cochrane review. ${ }^{7}$ The research proposal involves a collaborative approach between orthopaedic surgeons, infectious diseases physicians, infection control clinicians and clinical epidemiologists. This research is embedded within the healthcare system facilitating the direct translation of research evidence into clinical practice.

\section{Author affiliations}

${ }^{1}$ Department of Surgery, St Vincent's Hospital Melbourne, University of Melbourne, Melbourne, Victoria, Australia

${ }^{2}$ Department of Orthopaedic Surgery, St Vincent's Hospital Melbourne, Melbourne, Victoria, Australia

${ }^{3}$ Department of Infectious Diseases, Alfred Hospital, Melbourne, Victoria, Australia

${ }^{4}$ Department of Epidemiology and Preventive Medicine, Monash University, Melbourne, Victoria, Australia

${ }^{5}$ Department of Infectious Diseases, St Vincent's Hospital Melbourne, Melbourne, Victoria, Australia

Contributors All authors have contributed significantly to the study design, statistical analysis, ethics and funding submissions and the writing of the protocol.

Funding This work was supported by the National Health and Medical Research Council (NHMRC) of Australia, grant number APP1057736.
Competing interests TNP is supported by an NHMRC Early Career Australian Clinical Fellowship (APP1069374). MMD is supported by an NHMRC Early Career Australian Clinical Fellowship (APP1035810). ACC is supported by an NHMRC Career Development Fellowship 2 (APP1068732).

Ethics approval The study design and protocol were reviewed and approved by the St Vincent's Hospital Human Research Ethics Committee (HREC-A 016/14 10 March 2014)

Provenance and peer review Not commissioned; peer reviewed for ethical and funding approval prior to submission.

Open Access This is an Open Access article distributed in accordance with the Creative Commons Attribution Non Commercial (CC BY-NC 3.0) license, which permits others to distribute, remix, adapt, build upon this work noncommercially, and license their derivative works on different terms, provided the original work is properly cited and the use is non-commercial. See: http:// creativecommons.org/licenses/by-nc/3.0/

\section{REFERENCES}

1. Berbari EF, Hanssen AD, Duffy MC, et al. Risk factors for prosthetic joint infection: case-control study. Clin Infect Dis 1998;27:1247-54.

2. Surin VV, Sundholm K, Backman L. Infection after total hip replacement. With special reference to a discharge from the wound. J Bone Joint Surg 1983;65-Br:412-18.

3. Peel TN, Dowsey MM, Daffy JR, et al. Risk factors for prosthetic hip and knee infections according to arthroplasty site. J Hosp Infect 2011;79:129-33.

4. Peel TN, Dowsey MM, Buising KL, et al. Cost analysis of debridement and retention for management of prosthetic joint infection. Clin Microbiol Infect 2013;19:181-6.

5. Whitehouse JD, Friedman ND, Kirkland KB, et al. The impact of surgical-site infections following orthopaedic surgery at a community hospital and a university hospital: adverse quality of life, excess length of stay and extra cost. Infect Control 2002;23:183-9.

6. Kirkland KB, Briggs JP, Trivette SL, et al. The impact of surgical-site infections in the 1990s: attributable mortality, excess length of hospitalization, and extra costs. Infect Control Hosp Epidemiol 1999;20:725-30.

7. Edwards PS, Lipp A, Holmes A. Preoperative skin antiseptics for preventing surgical wound infections after clean surgery. Cochrane Database Syst Rev 2004;(3):CD003949.

8. Mangram AJ, Horan TC, Pearson ML, et al. Guideline for prevention of surgical site infection, 1999. Hospital Infection Control Practices Advisory Committee. Infect Control Hosp Epidemiol 1999;20: 250-78.

9. Dohmen PM. Antibiotic resistance in common pathogens reinforces the need to minimise surgical site infections. J Hosp Infect 2008;70 (Suppl 2):15-20.

10. Fishman N. Antimicrobial stewardship. Am J Med 2006;119(6 Suppl 1):S53-61.

11. Paterson DL. Resistance in gram-negative bacteria: enterobacteriaceae. Am J Med 2006;119(6 Suppl 1):S20-8d.

12. Fredricks DN. Microbial ecology of human skin in health and disease. J Investig Dermatol Symp Proc 2001;6:167-9.

13. Krizek TJ, Robson MC. Evolution of quantitative bacteriology in wound management. Am J Surg 1975;130:579-84.

14. Southwood RT, Rice JL, McDonald PJ, et al. Infection in experimental hip arthroplasties. J Bone Joint Surg 1985;67-Br:229-31.

15. Maiwald M, Chan ES. The forgotten role of alcohol: a systematic review and meta-analysis of the clinical efficacy and perceived role of chlorhexidine in skin antisepsis. PLoS ONE 2012;7:e44277.

16. Darouiche RO, Wall MJ Jr, Itani KM, et al. Chlorhexidine-alcohol versus povidone-iodine for surgical-site antisepsis. $N$ Engl J Med 2010;362:18-26.

17. NHMRC. Australian guidelines for the prevention and control of infection in healthcare: commonwealth of Australia. National Institute for Health and Clinical Excellence, 2010.

18. National Institute for Health and Clinical Excellence. Surgical site infection prevention and treatment of surgical site infection. London: RCOG Press at the Royal College of Obstetricians and Gynaecologists, 2008.

19. AORN. Recommended practices for preoperative patient skin antisepsis standards, recommended practices, and guidelines. New York: AORN, 2013.

20. Ingi Lee MM, Rajender K, Agarwal MM, et al. Systematic review and cost analysis comparing use of chlorhexidine with use of iodine for 
preoperative skin antisepsis to prevent surgical site infection. Infect Control Hosp Epidemiol 2010;31:1219-29.

21. Adams RJ, Tucker G, Hugo G, et al. Projected future trends of hospital service use for selected obesity-related conditions. Obes Res Clin Pract 2008;2:133-41.

22. Australian Orthopaedic Association. National Bone and Joint Registry Annual Report. 2012.

23. Caroll K, Dowsey M, Choong P, et al. Risk factors for superficial wound complications in hip and knee arthroplasty. Clin Microbiol Infect 2014;20:130-5.

24. Dowsey MM, Kilgour ML, Santamaria NM, et al. Clinical pathways in hip and knee arthroplasty: a prospective randomised controlled study. Med J Aust 1999;170:59-62.

25. Schulz KF, Altman DG, Moher D. CONSORT 2010 statement: updated guidelines for reporting parallel group randomized trials. Ann Intern Med 2010;152:726-32.

26. Horan TC, Gaynes RP, Martone WJ, et al. CDC definitions of nosocomial surgical site infections, 1992: a modification of CDC definitions of surgical wound infections. Infect Control Hosp Epidemiol 1992;13:606-8.

27. Wilson AP, Treasure T, Sturridge MF, et al. A scoring method (ASEPSIS) for postoperative wound infections for use in clinical trials of antibiotic prophylaxis. Lancet 1986;1:311-13.

28. Dindo D, Demartines N, Clavien PA. Classification of surgical complications: a new proposal with evaluation in a cohort of 6336 patients and results of a survey. Ann Surg 2004;240:205-13.
29. Parvizi J, Zmistowski B, Berbari E, et al. New definition for periprosthetic joint infection: from the workgroup of the musculoskeletal infection society. Clin Orthop Relat Res 2011;469:2992-4.

30. Marculescu CE, Cantey JR. Polymicrobial prosthetic joint infections. Clin Orthop Relat Res 2008;466:1397-404.

31. Mirra JM, Amstutz HC, Matos M, et al. The pathology of the joint tissues and its clinical relevance in prosthesis failure. Clin Orthop Relat Res 1976;117:221-40.

32. Trampuz A, Hanssen AD, Osmon DR, et al. Synovial fluid leukocyte count and differential for the diagnosis of prosthetic knee infection. Am J Med 2004;117:556-62.

33. Cook J, Bruckner T, MacLennan G, et al. Clustering in surgical trials - database of intracluster correlations. Trials 2012;13:2.

34. Campbell MK, Mollison J, Steen N, et al. Analysis of cluster randomized trials in primary care: a practical approach. Fam Pract 2000;17:192-6.

35. Hemming K, Girling AJ, Sitch AJ, et al. Sample size calculations for cluster randomised controlled trials with a fixed number of clusters. BMC Med Res Methodol 2011:11:102.

36. Lilford RJ, Pauker SG, Braunholtz DA, et al. Decision analysis and the implementation of research findings. BMJ 1998;317: 405-9.

37. Briggs A, Sculpher M. An introduction to Markov modelling for economic evaluation. Pharmacoeconomics 1998:13:397-409.

38. Wesley D. Life table analysis. J Insur Med 1998;30:247-54. 